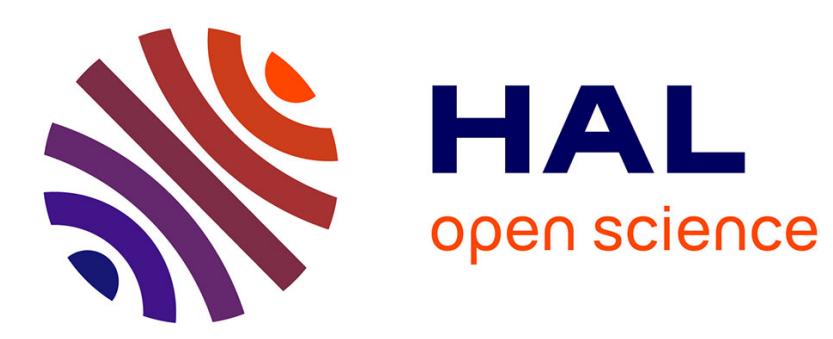

\title{
Almost periodic motion of a string vibrating against a straight fixed obstacle Alain Haraux, Henri Cabannes
}

\section{To cite this version:}

Alain Haraux, Henri Cabannes. Almost periodic motion of a string vibrating against a straight fixed obstacle. Nonlinear Analysis: Theory, Methods and Applications, 1983, 7, pp.129-141. 10.1016/0362546X(83)90076-7 . hal-01527087

\section{HAL Id: hal-01527087 \\ https://hal.science/hal-01527087}

Submitted on 23 May 2017

HAL is a multi-disciplinary open access archive for the deposit and dissemination of scientific research documents, whether they are published or not. The documents may come from teaching and research institutions in France or abroad, or from public or private research centers.
L'archive ouverte pluridisciplinaire HAL, est destinée au dépôt et à la diffusion de documents scientifiques de niveau recherche, publiés ou non, émanant des établissements d'enseignement et de recherche français ou étrangers, des laboratoires publics ou privés.

\section{(c)(1)}

Distributed under a Creative Commons Attribution| 4.0 International License 


\title{
ALMOST PERIODIC MOTION OF A STRING VIBRATING AGAINST A STRAIGHT FIXED OBSTACLE
}

\author{
A. Haraux* ànd H. Cabannes $\dagger$ \\ Laboratoires d'Analyse Numérique et Fonctionnelle* et de Mécanique Théoriquet, Université Pierre et Marie \\ Curie, 4 Place Jussieu, 75005 Paris, France
}

Key words and phrases: Almost periodic motion, vibrating string.

\section{INTRODUCTION}

IN A PLANE with normalized coordinate system $0 x u$, we consider the small oscillations of a vibrating string with fixed ends $( \pm 1 / 2,0)$. The free oscillations of this string are perturbed by the presence of a fixed obstacle $\{u=-h\}$, against which it rebounds following a law which insures conservation of the energy $(0 \leqslant h<1)$.

At time $t=0$, the string is at rest in a position $u(x, 0)=u_{0}(x)$ such that

$$
\begin{aligned}
& u_{0} \in H_{0}^{1}(]-1 / 2,1 / 2[) \\
& 0 \leqslant u_{0} \leqslant 1 \quad \text { on }[-1 / 2,1 / 2] .
\end{aligned}
$$

There exists $a, b$ in $]-1 / 2,1 / 2[, a \leqslant b$ such that

$$
\left.\begin{array}{l}
u_{0} \text { is non-decreasing and } u_{0}(x)<1 \text { on }[-1 / 2, a[ \\
u_{0}(x)=1 \text { for } x \in[a, b] \\
\left.\left.u_{0} \text { is non-increasing and } u_{0}(x)<1 \text { on }\right] b, 1 / 2\right] .
\end{array}\right\}
$$

If there was no obstacle, the motion of the string would be described by the wave equation:

$$
\square u=\frac{\partial^{2} u}{\partial t^{2}}-\frac{\partial^{2} u}{\partial x^{2}}=0,
$$

a classical approximation following d'Alembert [1] for the small (2-periodic) oscillations around $u=0$.

In case there is an obstacle, Amerio \& Prouse [2, linear obstacle], then Schatzman [12, concave obstacle] studied a nonlinear version of (4), assuming that there is no longitudinal perturbation and no energy loss during the shocks. Following [8] and setting $\Omega=]-1 / 2,1 / 2[$, one looks for solutions in the functional class

$$
u \in C\left(R^{+}, H_{0}^{1}(\Omega)\right) \cap W^{1, \infty}\left(R^{+}, L^{2}(\Omega)\right)
$$

satisfying the system

$$
\begin{aligned}
& u \geqslant-h \quad \text { in } R^{+} \times \Omega \\
& \operatorname{Supp}(\square u) \subset\{(x, t), u(x, t)>-h\}
\end{aligned}
$$




$$
\frac{\partial}{\partial x}\left\{-2 \frac{\partial u}{\partial t} \cdot \frac{\partial u}{\partial x}\right\}+\frac{\partial}{\partial t}\left\{\left(\frac{\partial u}{\partial t}\right)^{2}+\left(\frac{\partial u}{\partial x}\right)^{2}\right\}=0 .
$$

Condition (8), which is a strengthened version of energy conservation, must hold in the sense of $\mathscr{D}^{\prime}(] 0,+\infty[\times \Omega)$. It implies that the energy integral

$$
\int_{\Omega}\left(\frac{\partial u}{\partial x}\right)^{2}+\left(\frac{\partial^{+} u}{\partial t}\right)^{2} \mathrm{~d} x=\int_{\Omega}\left(\frac{\partial u}{\partial x}\right)^{2}+\left(\frac{\partial^{-} u}{\partial t}\right)^{2} \mathrm{~d} x
$$

remains unchanged through the motion.

Notice that in case a shock takes place simultaneously along a set of non-zero measure in $\Omega$, we cannot have

$$
\frac{\partial u}{\partial t} \in C\left(R^{-}, L^{2}(\Omega)\right) .
$$

Schatzman studied the existence and uniqueness of a solution of $(S)$ such that

$$
u(x, 0)=u_{0}(x), \frac{\partial^{+} u}{\partial t}(x, 0)=v_{0}(x) .
$$

Actually, the existence and uniqueness of a solution for $(S)+(9)$ is obtained in the functional class (5). The solution constructed satisfies the additional property:

$$
\square u \geqslant 0 \quad \text { in } \mathscr{D}^{\prime}(] 0,+\infty[\times \Omega)
$$

which is used to get uniqueness, and is interpreted in [9] as equivalent to subsonic propagation of interactions.

In our case, since $\partial u / \partial t(x, 0)=0$, we look for solutions (even as a function of $t$ ) defined on $R \times \bar{\Omega}$. Our purpose is to give a precise meaning to the physical idea of 'vibrations' against the obstacle.

We prove that the solution is strongly almost-periodic as a function from $R$ to $H_{0}^{1}(\Omega)$, generally not exactly periodic in $t$. We also sketch out some simple results concerning the non-harmonic Fourier series of $u(x, t)$ with respect to $t$. Further computations in this direction are planned for the future.

\section{STATEMENT OF THE RESULTS}

Let us denote by $(\Sigma)$ the system $(5) \rightarrow(10)$ with $v_{0}=0$ and $R^{+}$replaced by $R$ everywhere. We recall $0 \leqslant h<1$.

THEOREM 1.1. If $u_{0}$ satisfies (1)-(3), the solution of $(\Sigma)$ is such that $u(t)-u(., t)$ is strongly almost-periodic as a function from $R$ to $H_{0}^{1}(\Omega)$. Moreover, the map $u_{0} \rightarrow u(t)$ is Lipschitzcontinuous from $H_{0}^{1}(\Omega)$ to $L^{\infty}\left(R, H_{0}^{1}(\Omega)\right)$.

Remark. In contrast with the case of equation (4), $u(t)$ is generally not time-periodic. More precisely, we have:

THEOREM 1.2. (a) $h=p / q$ with $p, q$ integers, the motion is periodic with $p+q$ as a period if $p+q$ is even, and $2(p+q)$ as a period if $p+q$ is odd. In the special case when $u_{0}(-x)=$ $u_{0}(x)$, we have always the period $p+q$.

In some cases the smallest period is smaller. 
(b) If $h \notin Q$, the motion is never periodic, except in the single case $u_{0}(x)=1-2|x|$, where the motion has the period $1+h$.

Remark. The aim of this paper is to give complete proofs of the results announced in [7], together with some more information.

Since $u$ is almost-periodic with respect to $t$, for every $\lambda \in R$, the limit

$$
\lim _{t \rightarrow+\infty} \frac{1}{2 t} \int_{-t}^{t} u(x, \theta) \mathrm{d} \theta=\hat{u}(x, \lambda)
$$

exists. The set $\mathscr{E}(u)=\{\lambda \in R, \hat{u}(\cdot, \lambda) \neq 0\}$ is called the set of exponents of $u$, and in general (cf. [4]) is denumerable. Here, we have a more precise result.

THEOREM 1.3. The exponents of $u$ lie in the additive subgroup of $R$ generated by $\pi$ and $2 \pi / 1+h=\omega$.

More precisely:

(a) For general $u_{0}$ satisfying (1)-(3), we have

$$
\mathscr{E}(u) \subset Z \pi+Z \omega .
$$

(b) If $u_{0}$ is even, the motion of $u(0, t)$ can be developed as a generalized Fourier series:

$$
u(0, t)=\lim _{N \rightarrow \infty}\left[\frac{1-h}{2}+\sum_{p=1}^{N} \sum_{k=-\infty}^{k=+\infty} \lambda_{k}^{p} \cos \{(2 p+1) \omega+2 k \pi\} t\right]
$$

where the infinite sums on the right-hand side converge uniformly and the convergence with respect to $N$ is uniform for $t \in R$.

(c) In the special case $u_{0}(x)=1-2|x+\lambda \sin 2 \pi x|,|\lambda| \leqslant \frac{1}{2 \pi}$

$$
\begin{array}{ll}
\lambda_{k}^{P}=-\frac{8(1+h)}{(2 p+1)^{2} \pi^{2}} J_{k}[(2 p+1) \lambda \omega] & \text { for } k>0 \\
\lambda_{-k}^{P}=(-1)^{k} \lambda_{k} & \text { for } k>0
\end{array}
$$

and

$$
\lambda_{0}^{p}=-\frac{4(1+h)}{(2 p+1)^{2} \pi^{2}} J_{0}[(2 p+1) \lambda \omega] \dagger
$$

\section{SOME EXPLICIT FORMULAS}

a. Case $u_{0}(x)=1-2|x|$

The solution of $(\Sigma)$ is easy to describe. For $0 \leqslant t \leqslant t^{*}=\frac{1+h}{2}$, the motion is given by

$$
\bar{u}(x, t)= \begin{cases}1-2 \sup \{|x|, t\} & \text { if } 0 \leqslant t \leqslant 1 / 2 \\ 1-2 \inf \{1-|x|, t\} & \text { if } 1 / 2 \leqslant t \leqslant \frac{1+h}{2} .\end{cases}
$$

\footnotetext{
$\dagger$ Here $J_{k}$ is Bessel's function of order $k$.
} 
At time $t^{*}=1+h / 2$, the string hits the obstacle $\{u=-h\}$ along the line segment $\{u=-h$, $|x| \leqslant(1-h) / 2\}$, with a uniform velocity $v\left(t^{*}\right)=v^{-}=-2$.

Then the velocity is reversed into $v^{+}=-v^{-}=+2$, and the motion proceeds backwards:

$$
\tilde{u}(x, t)=\tilde{u}(x, 1+h-t), \forall t \in\left[\frac{1+h}{2}, 1+h\right] .
$$

Thus $\partial \tilde{u} / \partial t(x, t)$ is discontinuous at $t=t^{*}$, while $\tilde{u}(x, t)$ remains continuous with respect to $t \in R$. The motion $\bar{u}$ is periodic in $t$ with period $1+h$.

Conditions (5) and (6) are easy consequences of (15), (16) as soon as (8) is checked (to insure strong continuity in $\left.H_{0}^{1}(\Omega)\right)$. It is obvious that $\tilde{u} \in W^{1, \infty}(R \times \Omega)$ and moreover, almost everywhere on $R \times \Omega$, we have

$$
\frac{\partial \tilde{u}}{\partial t} \cdot \frac{\partial \tilde{u}}{\partial x}=0,\left(\frac{\partial \tilde{u}}{\partial t}\right)^{2}+\left(\frac{\partial \tilde{u}}{\partial x}\right)^{2}=4 .
$$

Then (8) follows as an immediate consequence.

Finally, a lengthy but standard computation gives the expression of $\square \tilde{u}$ : for every $\varphi \in$ $\mathscr{D}(R \times \Omega)$, we have

$$
\langle\square \tilde{u}, \varphi\rangle=4 \sum_{m \in Z} \int\left\{|x| \leqslant \frac{1-h}{2}\right\} \varphi\left[x,(2 m+1) \frac{1+h}{2}\right\rceil \mathrm{d} x .
$$

This formula implies (7) and (10).

b. The 'regular' case

In addition to (1)-(3), we assume now

$$
\left.\begin{array}{l}
u_{0} \in C^{2}([-1 / 2,1 / 2]), \text { and } u_{0}^{\prime \prime}(-1 / 2)=u_{0}^{\prime \prime}(1 / 2)=0 \\
u_{0}^{\prime}(a)=u_{0}^{\prime}(b)=u_{0}^{\prime \prime}(a)=u_{0}^{\prime \prime}(b)=0 .
\end{array}\right\}
$$

In order to compute $u(x, t)$, we generalize an idea used in [11] by Reder. We define a function $F: R \rightarrow R$ in several steps.

Step 1. If $x \in[-1 / 2,1 / 2]$, we set

$$
F(x)= \begin{cases}\frac{u_{0}(x)-1}{2} & \text { if }-1 / 2 \leqslant x \leqslant a \\ 0 & \text { if } a \leqslant x \leqslant b \\ \frac{1-u_{0}(x)}{2} & \text { if } b \leqslant x \leqslant 1 / 2 .\end{cases}
$$

It is clear from (19) that $F \in C^{2}([-1 / 2,1 / 2])$.

Step 2. If $t \in[1 / 2,3 / 2]$, we define

$$
F(t)=1-F(1-t) .
$$


It is clear that $F \in C^{2}([1 / 2,3 / 2])$. Since $F^{\prime \prime}(1 / 2)=0$, we have

$$
F \in C^{2}([-1 / 2,3 / 2])
$$

Step 3. We extend $F$ on $R$ by the condition

$$
\forall t \in R, F(t+2)=F(t)+2 .
$$

We introduce $f(t)=F(t)-t$ : then $f(t)$ is 2-periodic on $R$.

We claim that $f$ and $F$ are in $C^{2}(R)$, because $F^{\prime}(-1 / 2)=F^{\prime}(3 / 2)$ and $F^{\prime \prime}(-1 / 2)=F^{\prime \prime}(3 / 2)$.

The first condition is a consequence of $(21)$, the second one follows from $F^{\prime \prime}(-1 / 2)=0$.

Some other properties of $F$ are summarized below.

LEMMA 3.1. $F$ is nondecreasing: $R \rightarrow R$. In addition (21) holds for $t \in R$, and we have also $F(t)=-1-F(-1-t), \forall t \in R$.

For every $x \in \bar{\Omega}$ and $t \in R$, we have

$$
-1 \leqslant F(x+t)+F(x-t) \leqslant 1
$$

Proof. Formula (21) means that for every $\theta \in[-1 / 2,3 / 2]$, we have

$$
F(\theta)+F(1-\theta)=1 \text {. }
$$

Given $t \in R$, we choose $m \in Z$ and $\theta \in[-1 / 2,3 / 2]$ such that $t=2 m+\theta$. Then:

$$
F(t)+F(1-t)=F(\theta)+F(1-\theta)=1 \text {. }
$$

Also, for $t \in R$, we have:

$$
F(t)+F(-1-t)=F(t)+F(1-t)-2=-1 .
$$

Let $x \leqslant 1 / 2$ : by $(21)$, we have

$$
F(x+t)+F(x-t)=F(x+t)-F(-x+t+1)+1 .
$$

But $x \leqslant 1 / 2 \Rightarrow-x+t+1 \geqslant x+t$.

Thus we obtain: $F(x+t)+F(x-t) \leqslant 1$.

Also, if $x \geqslant-1 / 2$, we write

$$
F(x+t)+F(x-t)=F(x-t)-F(-1-x-t)-1 \geqslant-1,
$$

since $x-t \geqslant-1-x-t$.

Since $-1 / 2 \leqslant F(x+t)+F(x-t) / 2 \leqslant 1 / 2$, we introduce:

$$
u(x, t)=\tilde{u}\left\{\frac{F(x+t)+F(x-t)}{2}, \frac{F(x+t)-F(x-t)}{2}\right\}
$$

for $(x, t) \in \bar{\Omega} \times R$.

LEMMA 3.2. $u$ is the solution of $(\Sigma)$ with initial conditions $\left(u_{0}, 0\right)$, and we have

$$
\sup _{t \in R}\left|u(., t)-u^{\prime}(., t)\right|_{H^{1}(\Omega)} \leqslant C\left|u_{0}-u_{0}^{\prime}\right|_{H^{1}(\Omega)}
$$

for $u_{0}, u_{0}^{\prime}$ 'regular' satisfying (1-3). 
Proof. Let us introduce for convenience

$$
X=\frac{F(x+t)+F(x-t)}{2}, \quad T=\frac{F(x+t)-F(x-t)}{2} .
$$

Then, in the sense of $\mathscr{D}^{\prime}(\Omega \times R)$, we have

$$
\left.\begin{array}{l}
\frac{\partial u}{\partial x}=\frac{\partial \tilde{u}}{\partial X} \cdot \frac{F^{\prime}(x+t)+F^{\prime}(x-t)}{2}+\frac{\partial \tilde{u}}{\partial T} \cdot \frac{F^{\prime}(x+t)-F^{\prime}(x-t)}{2} \\
\frac{\partial u}{\partial t}=\frac{\partial \tilde{u}}{\partial X} \cdot \frac{F^{\prime}(x+t)-F^{\prime}(x-t)}{2}+\frac{\partial \bar{u}}{\partial T} \cdot \frac{F^{\prime}(x+t)+F^{\prime}(x-t)}{2}
\end{array}\right\}
$$

Using (18), we get immediately that $\partial u / \partial t$ and $\partial u / \partial x$ are in $L^{\infty}(\Omega \times R)$ and moreover:

$$
\left.\begin{array}{l}
\left(\frac{\partial u}{\partial x}\right)^{2}+\left(\frac{\partial u}{\partial t}\right)^{2}=2\left[F^{\prime 2}(x+t)+F^{\prime 2}(x-t)\right] \\
\frac{\partial u}{\partial x} \cdot \frac{\partial u}{\partial t}=F^{\prime 2}(x+t)-F^{\prime 2}(x-t)
\end{array}\right\}
$$

The properties (5) and (6) are immediately checked. On the other hand, in the sense of $\mathscr{D}^{\prime}(\Omega \times R)$, we obviously have

$$
\begin{aligned}
& \frac{\partial}{\partial t}\left\{\left(\frac{\partial u}{\partial x}\right)^{2}+\left(\frac{\partial u}{\partial t}\right)^{2}\right\}=4\left[F^{\prime}(x+t) F^{\prime \prime}(x+t)-F^{\prime}(x-t) F^{\prime \prime}(x-t)\right] \\
& \frac{\partial}{\partial x}\left(\frac{\partial u}{\partial x} \cdot \frac{\partial u}{\partial t}\right)=2\left[F^{\prime}(x+t) F^{\prime \prime}(x+t)-F^{\prime}(x-t) F^{\prime \prime}(x-t)\right]
\end{aligned}
$$

which implies (8).

Differentiating (25) in the distribution sense, we get after reduction:

$$
\square u(x, t)=F^{\prime}(x+t) F^{\prime}(x-t) \square \tilde{u}[X(x, t), T(x, t)],
$$

this formula making sense because $\square \tilde{u}$ is a measure and $F^{\prime}$ is continuous. From (27), we deduce that (7) and (10) are satisfied.

As a consequence of lemma 3.1 , we have for every $t \in R: X(1 / 2, t)=1 / 2$ and $X(-1 / 2, t)=-1 / 2$.

Thus $u(1 / 2, t)=0$ and $u(-1 / 2, t)=0$, and we have $u(t) \in H_{0}^{1}(\Omega)$ for all $t$.

To verify the initial condition, we remark that $u(x, 0)=\tilde{u}(F(x), 0)=\tilde{u}_{0}(F(x))=$ $1-2|\mathrm{~F}(\mathrm{x})|$ and $(20)$ implies $1-2|F(x)|=u_{0}(x)$ in $\bar{\Omega}$.

It is easy to check that

$$
\frac{u(x, t)-u_{0}(x)}{t} \rightarrow 0 \text { in } C(\tilde{\Omega}) \text { as } t \rightarrow 0, \text { which implies } \frac{\partial u}{\partial t}(x, 0)=0 .
$$

Finally, we can see that the mapping $u_{0} \rightarrow f$ is Lipschitzian from $H_{0}^{1}(\Omega)$ to $H^{1}([0,2])$. Since $\bar{u} \in W^{1, \infty}(\Omega \times R)$, the mapping:

$$
f \rightarrow u(., t) \text { is Lipschitz-continuous from } H^{1}([0,2]) \text { to } L^{\infty}\left(R, H^{1}(\Omega)\right) .
$$

Combining these two remarks, we get (24), which will appear a convenient tool to treat the more general case where $u_{0}$ is only in $H_{0}^{1}(\Omega)$. 


\section{c. The general case}

If $u_{0}$ satisfies conditions (1-3), we can still define $F(t)$ as in the paragraph above. Then $F(t)$ is non-decreasing, in $H^{\mathrm{l}}(R)$ and $f(t)=F(t)-t$ is 2-periodic.

We compute $u(x, t)$ by means of formula (23). To check that $u(x, t)$ is the solution of $(\Sigma)$ starting from $\left(u_{0}, 0\right)$, we can choose a sequence of initials $u_{0}^{n}$ satisfying (19) with $u_{0}^{n} \rightarrow u_{0}$ in $H_{0}^{1}(\Omega)$. According to $(24), u^{n}(x, t)$ will converge to $u(x, t)$ in $L^{x}\left(R, H_{0}^{1}(\Omega)\right)$. A similar calculation shows that $\partial u^{n} / \partial t$ converges to $\partial u / \partial t$ in $L^{\infty}\left(R, L^{2}(\Omega)\right)$.

Thus all conditions $(5) \rightarrow(10)$ are checked by density.

\section{PROOF OF THEOREM 1.1}

Because of (24), it is enough to check the almost-periodicity when $u_{0}$ is 'regular', so that $F \in C^{2}(R)$.

a. As a first step, we prove that $t \rightarrow u(., t)$ is almost-periodic in $C(\bar{\Omega})$, by using directly Bochner's criterion.

Let $\left(t_{n}\right)$ be any sequence of real numbers. We write:

$$
\begin{array}{ll}
t_{n}=2 m_{n}+\rho_{n} & \rho_{n} \in[0,2] \\
2 m_{n}=k_{n}(1+h)+\sigma_{n} & \sigma_{n} \in[0,1+h]
\end{array}
$$

where $m_{n}$ and $k_{n}$ are in $Z$. Thus

$$
u\left(x, t+t_{n}\right)=\tilde{u}\left\{\frac{F\left(x+t+\rho_{n}\right)+F\left(x-t-\rho_{n}\right)}{2}, \frac{F\left(x+t+\rho_{n}\right)-F\left(x-t-\rho_{n}\right)}{2}+\sigma_{n}\right\}
$$

We may assume $\lim _{n \rightarrow \infty} \rho_{n_{k}}=\rho$ and $\lim _{n \rightarrow \infty} \sigma_{n_{k}}=\sigma$, by extracting a subsequence of $\left(t_{n}\right)$. Then, because of the uniform continuity of $F$ and $\tilde{u}$ the sequence $u\left(x, t+t_{n_{k}}\right)$ converges uniformly on $\bar{\Omega} \times R$ to

$$
u^{*}(x, t)=\tilde{u}\left\{\frac{F(x+t+\rho)+F(x-t-\rho)}{2}, \frac{F(x+t+\rho)-F(x-t-\rho)}{2}+\sigma\right\} .
$$

\section{b. Precompactness of the range of $u$ in $H_{0}^{1}(\Omega)$}

Thanks to conservation of energy, $u(t)$ is bounded in $H_{0}^{1}(\Omega)$, thus weakly almost-periodic in $H_{0}^{\mathrm{l}}(\Omega)$. According to [10], Theorem 2.11, the strong almost-periodicity is equivalent to precompactness of the range in $H_{0}^{1}(\Omega)$. Even in the 'regular' case, it is not so easy to check because we cannot approach $u$ in $L^{\infty}\left(R, H_{0}^{1}(\Omega)\right)$ by regular functions since $\tilde{u}$ has discontinuous derivatives. We need a technical lemma.

LEMMA 4.1. With the notations of (a), there exists a denumerable set $S \subset[-1 / 2,1 / 2]$ such that

$$
x \notin S \Rightarrow \lim _{k \rightarrow \infty} \frac{\partial u}{\partial x}\left(x, t_{n_{k}}\right)=\frac{\partial u^{*}}{\partial x}(x, 0) .
$$

Proof. Let us use the notations

$$
X(x, t)=\frac{F(x+t)+F(x-t)}{2}, T(x, t)=\frac{F(x+t)-F(x-t)}{2}
$$




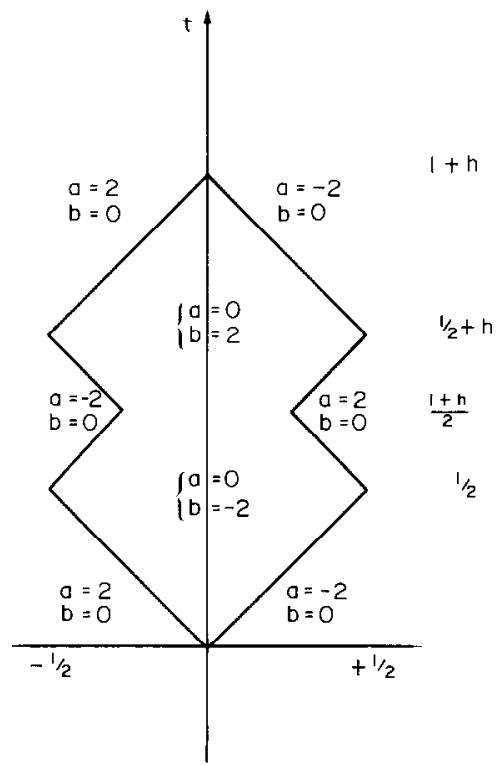

Fig. 1.

$$
\begin{array}{ll}
a(x, t)=\frac{\partial \tilde{u}}{\partial x}(x, t) & (\text { for all } t \text { and almost every } x) \\
b(x, t)=\frac{\partial \tilde{u}}{\partial t}(x, t) & (\text { for all } x \text { and almost every } t) .
\end{array}
$$

The functions $a, b$ take only the values $0,-2$ and +2 , their discontinuities lie on some curves of $\bar{\Omega} \times R$.

The periodicity of $\tilde{u} / t$ allows us to draw a picture in $\bar{\Omega} \times[0,1+h]$ (cf. Fig. 1). For $x \in$ $[-1 / 2,1 / 2[$ and $0<\delta<1 / 2-x$, we define

$$
\begin{aligned}
& \alpha(x, t, \delta)=1 / \delta\{\tilde{u}[X(x+\delta, t)-T(x+\delta, t)]-\tilde{u}[X(x, t), T(x+\delta, t)]\} \\
& \beta(x, t, \delta)=1 / \delta\{\tilde{u}[X(x, t), T(x+\delta, t)]-\tilde{u}[X(x, t), T(x, t)]\}
\end{aligned}
$$

Also, for $t$ fixed, we introduce two open sets

$$
\begin{gathered}
\omega=\{x \in]-1 / 2,+1 / 2\left[, \frac{\partial X}{\partial x}(x, t) \neq 0\right\} \\
\omega^{\prime}=\{x \in]-1 / 2,+1 / 2\left[, \frac{\partial T}{\partial x}(x, t) \neq 0\right\} .
\end{gathered}
$$

-First we notice that if $x \notin \omega$, then

$$
X(x+\delta, t)-X(x, t)=0(\delta) \quad \text { when } \delta \rightarrow 0
$$

Since $\tilde{u}$ is Lipschitzian in both variables, we conclude

$$
\lim _{\delta \rightarrow 0} \alpha(x, t, \delta)=0 \quad \text { in this case. }
$$


-Let $\omega_{n}$ be the sequence of connected components of $\omega$.

For every $n$, we have either $\partial X / \partial x(x, t)>0$ in $\omega_{n}$, or $\partial X / \partial x(x, t)<0$ in $\omega_{n}$. Thus, in each $\omega_{n}$, there are at most 2 values $\left(x_{n, k}\right) k \in\{1,2\}$ such that (a) is discontinuous at $\left[X\left(x_{n, k}, t\right), T\left(x_{n, k}, t\right)\right]$. As a consequence, if $x \in \omega \backslash \cup_{n, k}^{\cup}\left\{x_{n, k}\right\}$ we find

$$
\lim _{\delta \rightarrow 0} \alpha(x, t, \delta)=a[X, T] \frac{\partial X}{\partial x} .
$$

In the same way, we obtain that except for denumerable values of $x$, we have

$$
\lim _{\delta \rightarrow 0} \beta(x, t, \delta)=b[X, T] \frac{\partial T}{\partial x} .
$$

As a consequence, there exists $S_{t}$ denumerable such that, for every $x \notin S_{t}$ :

$$
\frac{\partial}{\partial x}[u(x, t)]=a[X, T] \frac{\partial X}{\partial x}+b[X, T] \frac{\partial T}{\partial x} .
$$

Let $S_{t_{n_{k}}}=S_{k}$. Then, for $x \notin \bigcup_{k \in N} S_{k}$, we have

$$
\forall k \in N, \frac{\partial}{\partial x}\left[u\left(x, t_{k}\right)\right]=a\left[X_{k}(x), T_{k}(x)\right] \frac{\partial X_{k}}{\partial x}+b\left[X_{k}(x), T_{k}(x)\right] \frac{\partial T_{k}}{\partial x}
$$

where $X_{k}(x)=X\left(x, t_{k}\right)$ and $T_{k}(x)=T\left(x, t_{k}\right)$. Finally, let

$$
\left\{\begin{array}{l}
X_{\rho}(x)=X(x, \rho) \\
T_{\rho, \sigma}(x)=T(x, \rho)+\sigma
\end{array}\right.
$$

By a very similar argument as above, we find $S_{\infty}$ denumerable in ]-1/2,1/2[ such that

$$
\begin{aligned}
x \notin \bigcup_{k \in N} S_{k}, x \notin S_{\infty} & \Rightarrow \lim _{k \rightarrow \infty} \frac{\partial}{\partial x}\left[u\left(x, t_{k}\right)\right] \\
& =a\left[X_{\rho}, T_{\rho, \sigma}\right] \frac{\partial X_{\rho}}{\partial x}+b\left[X_{\rho}, T_{\rho, \sigma}\right] \frac{\partial T_{\rho, \sigma}}{\partial x} .
\end{aligned}
$$

But, except for $x \in S^{*}$ denumerable, this is precisely equal to $\partial / \partial x\left[u^{*}(x, 0)\right]$. Finally, (29) holds with $S=S^{*} \cup S_{\infty \cup} \bigcup_{k \in N} S_{k}$.

Proposition 4.1. The range of $u(t)$ is precompact in $H_{0}^{1}(\Omega)$ ['regular' case].

Proof. Let $\left(t_{n}\right)$ be an arbitrary sequence of reals. There exists a subsequence $\left(t_{n_{k}}\right), \rho$ and $\sigma$ such that

$$
u\left(x, t_{n_{k}}\right) \rightarrow u^{*}(x, 0) \quad \text { in } C(\bar{\Omega}) .
$$

By lemma: $\partial u / \partial x\left(x, t_{n_{k}}\right) \rightarrow \partial u^{*} / \partial x(x, 0)$ a.e. in $\Omega$.

Since $\partial u / \partial x\left(x, t_{n_{k}}\right)$ remains bounded in $L^{\infty}(\Omega)$ by (30), Lebesgue's theorem implies

$$
\int_{\Omega}\left|\frac{\partial u}{\partial x}\left(x, t_{n_{k}}\right)-\frac{\partial u^{*}}{\partial x}(x, 0)\right|^{2} \mathrm{~d} x \underset{k \rightarrow \infty}{\rightarrow} 0 .
$$

Hence, $u\left(x, t_{n_{k}}\right) \rightarrow u^{*}(x, 0)$ in $H_{0}^{1}(\Omega)$. 
End of the proof of Theorem 1.1. In the regular case, theorem 1.1 is now an obvious consequence of (a), proposition 4.1 and $[10$, theorem $2.11, \mathrm{p} .48]$, applied with $Y=C=$ $C(\bar{\Omega})$ and $Z=H_{0}^{1}(\Omega)$.

In the general case, we can use (24) which shows that $u(x, t)$ is the limit in $L^{x}\left(R, H_{0}^{\mathrm{l}}(\Omega)\right)$ of solutions associated to 'regular' initials $u_{0}^{n}$. This finishes the proof of theorem 1.1, since the statement on $u_{0} \rightarrow u(t)$ is actually a consequence of (24).

\section{PROOF OF THFORFM 1.2}

(a) Since $F(t)-t$ is 2-periodic and $\tilde{u}(x, t)$ is $1+h$-periodic in $t$, the first assertion is an obvious consequence of formula (23).

If $u_{0}(-x)=u_{0}(x)$, then $b=-a$ and $F(t)$ is odd. Then $F(t)-t$ is 1-periodic, and the second assertion follows.

Actually, $F(t)=t+\alpha \sin 2 n \pi t$ is non-decreasing as soon as $2 n \pi|\alpha|<1$, and corresponds to the initial datum $u_{0}(x)=1-2|x+\alpha \sin 2 n \pi x|$. In this case, $F(t)-t$ is $1 / n$-periodic.

If for instance $h=1 / n$, then $(n+1) / n=1+h$ is a period for $u(., t)$, which is strictly (for $n \geqslant 2$ ) smaller than $n+1$. For $n=2 p$, we get a period smaller than $n+1=p+q$.

(b) If $u(., t)$ is periodic with a period $\tau$, then the functions

$$
g_{\varepsilon, \alpha, \beta}(t)=\int_{t}^{t+\varepsilon} \int_{\alpha}^{\beta}\left[\left(\frac{\partial u}{\partial x}\right)^{2}+\left(\frac{\partial u}{\partial \theta}\right)^{2}+2 \frac{\partial u}{\partial x} \cdot \frac{\partial u}{\partial \theta}\right] \mathrm{d} x \mathrm{~d} \theta
$$

are also $\tau$-periodic, independently of $\varepsilon, \alpha, \beta$ for $-\frac{1}{2} \leqslant \alpha<\beta \leqslant \frac{1}{2}$. As a consequence of (26),

$$
g_{\varepsilon, \alpha, \beta}(t)=4 \int_{t}^{t, \varepsilon} \int_{\alpha}^{\beta} F^{\prime 2}(x+\theta) \mathrm{d} x \mathrm{~d} \theta
$$

Now it is obvious that

$$
\lim _{\varepsilon \rightarrow 0} \frac{1}{4 \varepsilon} g_{\varepsilon, \alpha, \beta}(t)=\int_{\alpha}^{\beta} F^{\prime 2}(x+t) \mathrm{d} x=\int_{t+\alpha}^{t+\beta} F^{\prime 2}(\sigma) \mathrm{d} \sigma
$$

Thus for every $l \in[0,1]$, the function

$$
\varphi(l, t)=\int_{t}^{t+l} F^{2}(\sigma) \mathrm{d} \sigma \text { is } \tau \text {-periodic. }
$$

Now we distinguish two possible cases:

1st case: $\tau \notin Q$.

Since $F^{\prime}$ is 2 -periodic, we must have $\varphi(l, t)=\varphi(l)$ for $l \in[0,1]$. Since $\varphi$ is a continuous function of $l$, it is immediate to deduce

$$
\int_{t}^{t+l} F^{2}(\sigma) \mathrm{d} \sigma=k^{2} l, \quad k \geqslant 0
$$

Then at every Lebesgue's point of $F^{\prime 2}$, we must have $F^{\prime 2}(t)=k^{2}$. Since $F^{\prime} \geqslant 0$ a.e., this allows us to conclude that $F^{\prime} \equiv k$ a.e. As a consequence of (22), we must have $k=1$. Also by (21), $F(1 / 2)=1 / 2$.

Thus $F(t)=t$, and $u_{0}(x)=1-2|F(x)|$ implies $u_{0}(x)=1-2|x|$. 
2nd case: $\tau=p / q \in Q \backslash\{0\}$

Then $2 q \tau=2 p$ is also a period for $u(., t)$.

On the other hand, by (23), we have:

$$
u(x, t+2 p)=\tilde{u}\left\{\frac{F(x+t)+F(x-t)}{2}, \frac{F(x+t)-F(x-t)}{2}+2 p\right\} .
$$

Choosing $t=0$ and $x=a$ for instance, we get $u(a, 2 p)=\tilde{u}(0,2 p)=\tilde{u}(0,0)=1$. The only possibility is $2 p=m(1+h), m \in Z \backslash\{0\}$.

Thus $h=2 p / m-1 \in Q$.

\section{THE EXPONENTS OF $u(t)$. (PROOF OF THEOREM 1.3)}

(a) Let $\omega=2 \pi / 1+h$, we can write $\tilde{u}$ as a Fourier series in $t$ :

$$
\tilde{u}(x, t)=\sum_{p=0}^{\infty} a_{p}(x) \cos (p \omega t)
$$

Since $\partial \bar{u} / \partial t \in L^{\infty}(\bar{\Omega} \times R)$, this series is uniformly convergent on $\bar{\Omega} \times R$. The coefficients $a_{p}$ are $C^{1}$ functions on $\bar{\Omega}=[-1 / 2,+1 / 2]$ and can be computed easily. Thus we have:

$$
u(x, t)=\lim _{N \rightarrow+x} \sum_{p=0}^{N} a_{p}[X(x, t)] \cos [p \omega T(x, t)]
$$

uniformly on $\bar{\Omega} \times R$ and we just need to examine the exponents of each term

$$
a_{p}[X(x, t) \cos [p \omega T(x, t)] .
$$

By a density argument, it is sufficient to do this in the 'regular' case. Then $F(t)-t=f(t)$ is a $C^{1}$ function, periodic with period 2 , and we get a first development:

$$
\begin{aligned}
& a_{p}[X(x, t)]=\sum_{k=0}^{\infty} \alpha_{k}^{p}(x) \cos k \pi t, \text { with (for instance) } \\
& \sum_{k=0}^{\infty}\left|\alpha_{k}^{p}\right|_{\infty}<+\infty .
\end{aligned}
$$

As a second step, we may write:

$$
\cos [p \omega T(x, t)]=\cos p \omega\left[t+\frac{1}{2}(f(x+t)-f(x-t))\right]=\cos p \omega t \beta(x, t)-\sin p \omega t \gamma(x, t) .
$$

The functions $\beta$ and $\gamma$ are in $C^{1}(\bar{\Omega} \times R)$ and thus:

$$
\begin{aligned}
& \beta(x, t)=\sum\left[\beta_{k}^{p}(x) \cos k \pi t+\delta_{k}^{p}(x) \sin k \pi t\right] \\
& \gamma(x, t)=\sum\left[\gamma_{k}^{p}(x) \cos k \pi t+\varepsilon_{k}^{p}(x) \sin k \pi t\right]
\end{aligned}
$$

with

$$
\sum_{k=0}^{\infty}\left(\left|\beta_{k}^{p}\right|_{\infty}+\left|\gamma_{k}^{p}\right|_{\infty}+\left|\delta_{k}^{p}\right|_{\infty}+\left|\varepsilon_{k}^{p}\right|_{\infty}\right)<+\infty .
$$

Thus the Cauchy product of the two series defining $a_{p}[X(x, t)]$ and $\cos [p \omega T(x, t)]$ is absolutely 
convergent in $C(\bar{\Omega})$, and after reduction, we are left with a development of the type:

$$
a_{p}[X] \cos [p \omega t]=\sum_{k \in Z}\left[\lambda_{k}(x) \cos (p \omega t+k \pi t)+\mu_{k}(x) \sin (p \omega t+k \pi t)\right]
$$

which is strongly almost-periodic in $C(\bar{\Omega})$ with exponents contained in $\{p \omega\}+Z \pi$ in the $L^{2}(\Omega)$ sense. Thus the exponents of $u(., t)$ in the sense of $L^{2}(\Omega)$ are in $Z \omega+Z \pi$, and of course it is the same set of exponents than in $H_{0}^{1}(\Omega)$.

(b) In case $u_{0}$ is even and we take $x=0$, some simplifications occur in the previous computations. We have

$$
\left\{\begin{array}{l}
a_{0}(0)=\frac{1-h}{2} \\
a_{p}(0)=-4(1+h) \frac{\sin ^{2}(p / 2) \pi}{p^{2} \pi^{2}}
\end{array}\right.
$$

We also obtain

$$
\begin{aligned}
& \cos (p \omega f(t))=\sum_{k \geqslant 0} \beta_{k}^{p} \cos (2 k \pi t) \\
& \sin (p \omega f(t))=\sum_{k \geqslant 0} \alpha_{k}^{p} \sin (2 k \pi t)
\end{aligned}
$$

with

$$
\begin{aligned}
& \beta_{k}^{p}=2 \int_{0}^{1} \cos (p \omega f(t)) \cos (2 k \pi t) \mathrm{d} t, \beta_{0}^{p}=\int_{0}^{1} \cos (p \omega f(t)) \mathrm{d} t \\
& \alpha_{k}^{p}-2 \int_{0}^{1} \sin (p \omega f(t)) \sin (2 k \pi t) \mathrm{d} t
\end{aligned}
$$

and we deduce easily

$$
\begin{aligned}
& u(0, t)=\lim _{N \rightarrow \infty}\left[\frac{1-h}{2}-4(1+h) \Phi_{N}(t)\right], \text { where } \\
& \Phi_{N}(t)=\sum_{p=1}^{N} \frac{1}{(2 p+1)^{2} \pi^{2}} \sum_{k \in Z} A_{k}^{p} \cos \{[(2 p+1) \omega+2 k \pi] t\}
\end{aligned}
$$

and

$$
A_{k}^{p}= \begin{cases}\frac{1}{2}\left(\beta_{k}^{2 p+1}+\alpha_{k}^{2 p+1}\right) & \text { if } k>0 \\ \beta_{0}^{2 p+1} & \text { if } k=0 \\ \frac{1}{2}\left(\beta_{|k|}^{2 p+1}-\alpha_{|k|}^{2 p+1}\right) & \text { if } k<0\end{cases}
$$

(c) Case $u_{0}(x)=1-2|x+\lambda \sin 2 \pi x|$

Then $f(t)=\lambda \sin 2 \pi t$, and the condition $F^{\prime} \geqslant 0$ is equivalent to $|\lambda| \leqslant 1 / 2 \pi$. 
Setting $c_{p}=\frac{1}{2}(2 p+1) \lambda \omega$, we obtain

$$
\begin{array}{ll}
A_{k}^{p}=2 J_{k}[(2 p+1) \lambda \omega]=2 \sum_{r \geqslant 0} \frac{(-1)^{r}\left(c_{p}\right)^{2 r+k}}{r !(r+k) !} & \text { if } k>0 \\
A_{0}^{p}=J_{0}[(2 p+1) \lambda \omega]=\sum_{r \geqslant 0} \frac{(-1)^{r}\left(c_{p}\right)^{2 r}}{(r !)^{2}} & \text { if } k=0 \\
A_{k}^{p}=2(-1)^{k} J_{|k|}[(2 p+1) \lambda \omega]=2(-1)^{k} \sum_{r \geqslant 0} \frac{(-1)^{r}\left(c_{p}\right)^{2 r+|k|}}{r !(r+|k| !)} & \text { if } k<0 .
\end{array}
$$

\section{REFERENCES}

1. d'Alembert J., Opuscules mathématiques, David éditeur, Paris (1761),

2. Amerio L. \& Prouse G., Study of the motion of a string vibrating against an obstacle, Rc. Mat. Applic. 8, 563-585 (1975).

3. Bamberger A. \& Schatzman M., New results on the vibrating string with a continuous obstacle, MRC Technical Summary Report 2073, University of Wisconsin (1980).

4. Besicovitch A. S., Almost periodic functions, Cambridge University Press, Cambridge (1932).

5. Bochner S., A new approach to almost periodicity, Proc. natn. Acad. Sci. U.S.A. 48, 2039-2043 (1962).

6. Cabannes H., Mouvements périodiques d'une corde vibrante en présence d'un obstacle rectiligne $Z$. angew. Math Phys. 31, 473-482 (1980).

7. Cabannes H. \& Haraux A., Mouvements presque périodiques d'une corde vibrante en présence d'un obstacle rectiligne, C.r. hebd. Séanc. Acad. Sci., Paris 191A, 563-565 (1980).

8. Cabannes H. \& Haraux A., Mouvements presque périodiques d'une corde vibrante en présence d'un obstacle fixe, rectiligne ou ponctuel, Int. J. Nonlinear Mechanics, to appear.

9. Citrini C. \& d'Acunto B., Sur le choc de deux cordes, C.r. hebd. Séanc. Acad. Sci., Paris 298A, 5-7 (1979).

10. Dafermos C. M., Almost periodic processes and almost periodic solutions of evolution equations, Dynamical Systems, Proc. Univ. of Florida Int. Symp., Academic Press, New York (1977).

11. REDER C., Etude qualitative d'un problème hyperbolique avec contrainte unilatérale. Thèse de 3ème cycle, Université de Bordeaux (1979).

12. SCHATZMAN M., An hyperbolic problem of second order with unilateral constraints: the vibrating string with a concave obstacle, J. math. Analysis Applic. 73, 138-191 (1980). 